\title{
Heart rate variability and surface electromyography of trained cyclists at different cadences
}

\section{Variabilidade da frequência cardíaca e eletromiografia de superfície em ciclistas treinados em diferentes cadências}

\author{
Bruno Saraiva ${ }^{1}$, Ester Silva², Rodrigo Polaquini Simões², Ana Paula Urdiales Garcia², Fabrício \\ Augusto Menegon ${ }^{3}$, Daniel Iwai Sakabe², Rodrigo Lício Ortolan ${ }^{4}$, Luiz Eduardo Barreto \\ Martins $^{5}$, Lucien Oliveira ${ }^{2}$, Aparecida Maria Catai ${ }^{2 *}$
}

ORIGINAL ARTICLE | ARTIGO ORIGINAL

\begin{abstract}
The heart rate variability (HRV) and surface electromyography (sEMG) are important tools in the evaluation of cardiac autonomic system and neuromuscular parameters, respectively. The aim of the study was to evaluate the behavior of HRV and sEMG of the vastus lateralis in two exercise protocols on a cycle ergometer at 60 and $80 \mathrm{rpm}$. Eight healthy men cyclists who have trained for at least two years were evaluated. Reduction was observed followed by stabilization of RMSSD and SDNN indices of HRV $(p<0.05)$ along with increases in the amplitude of the sEMG signal $(p<0.05)$ in both protocols. Significant correlations were observed between the responses of HRV and sEMG in the cadence of $60 \mathrm{rpm}$ (RMSSD and sEMG: $\mathrm{r}=-0.42, \mathrm{p}=0.03$; SDNN and sEMG: $\mathrm{r}=-0.45, \mathrm{p}=0.01)$ and $80 \mathrm{rpm}$ (RMSSD and $\mathrm{sEMG:} \mathrm{r}=$ $-0.47, \mathrm{p}=0.02$; SDNN and sEMG: $\mathrm{r}=-0.49, \mathrm{p}=0.01$ ), yet no difference was observed for these variables between the two protocols. We concluded that the parasympathetic cardiac responses and sEMG are independent of cadences applied at the same power output.
\end{abstract}

Keywords: heart rate variability, surface electromyography, exercise

RESUMO

A variabilidade da frequência cardíaca (VFC) e a eletromiografia de superfície (EMGs) são ferramentas importantes na avaliação do sistema autonômico cardíaco e dos parâmetros neuromusculares respetivamente. O objetivo do estudo foi avaliar o comportamento da VFC e da EMGs do músculo vasto lateral em dois protocolos de exercício no ciclo ergómetro em 60 e $80 \mathrm{rpm}$. Oito homens ciclistas treinados há pelo menos 2 anos foram avaliados. Foi verificada redução dos índices RMSSD e SDNN da VFC seguida por estabilização $(p<0.05)$ e aumento da amplitude do sinal da EMGs $(p<0.05)$ em ambos os protocolos. Correlações significativas foram observadas entre as respostas da VFC e da EMGs na cadência de $60 \mathrm{rpm}$ (RMSSD e EMGs: $\mathrm{r}=-0.42$, $\mathrm{p}=0.03$; SDNN e EMGs: $\mathrm{r}=-0.45, \mathrm{p}=0.01$ ) e 80 rpm (RMSSD e EMGs: $\mathrm{r}=$ -0.47, $\mathrm{p}=0.02$; SDNN e EMGs: $\mathrm{r}=-0.49, \mathrm{p}=0.01$ ), e não houve diferença destas variáveis entre os dois protocolos. Concluímos que as respostas parassimpática cardíaca e da EMGs são independentes das cadências aplicadas na mesma intensidade do esforço.

Palavras-chave: Variabilidade da frequência cardíaca, eletromiográfia de superfície, exercício físico

\footnotetext{
Manuscript received at July $25^{\text {th }} 2014$; Accepted at October $30^{\text {th }} 2015$

${ }^{1}$ Study Group in Exercise Physiology of Federal University of Rondônia, Porto Velho, Rondônia, Brazil

2 Department of Physiotherapy, Cardiovascular Physiotherapy Laboratory, Nucleus of Research in Physical Exercise, Federal University of Sao Carlos, Sao Carlos, Sao Paulo, Brazil

${ }^{3}$ Department of Public Health, Federal University of Santa Catarina, Florianópolis, Santa Catarina, Brazil.

${ }^{4}$ Federal Institute of Education, Science and Technology South of Minas Gerais, Poços de Caldas, Minas Gerais, Brazil.

${ }^{5}$ Laboratory of Exercise Physiology, Faculty of Physical Education, State University of Campinas, Campinas, SP, Brazil

* Corresponding author. Departamento de Fisioterapia, Laboratório de Fisioterapia Cardiovascular, Universidade Federal de São Carlos, Rodovia Washington Luis, km 235, CEP: 13565-905, São Carlos, São Paulo, Brazil E-mail: mcatai@ufscar.br
} 


\section{INTRODUCTION}

Physical exercise promotes physiological adjustments in various systems within the body including the neuro-muscular, cardiopulmonary, thermoregulatory and neuro-endocrine systems (Ribeiro et al., 2000) in an attempt to maintain homeostasis of the system with the largest demand caused by working muscles during exercise (Silva et al., 1999). For example, the performance of the cardiac autonomic nervous system by sympathetic and parasympathetic components are fundamental to appropriate adjustments of the cardiovascular system with the onset of physical activity. Such adjustments can be evaluated by analyzing the periodic variation of R-R intervals between consecutive heart beats, called heart rate variability (HRV) (Longo, Ferreira, \& Correia, 1995).

Thus HRV is an important tool that can be used during exercise to assess the parameter settings of the cardiac autonomic system and through the components of HRV is possible to determine the anaerobic threshold (AT) during exercise and consequently relate to the aerobic capacity of the individual (Di Michele et al., 2012; Marães et al., 2005; Sales et al., 2011; Seluyanov, Kalinin, Pack, Mayevskaya, \& Konrad, 2011; Takahashi et al., 2005). The advantages of HRV include being a non-invasive procedure with a low proportion of errors, using relatively simple equipment at low cost (Catai et al., 2002; Simões et al., 2014).

Nevertheless, other noninvasive parameters also have been utilized providing significant information related to exercise including: surface electromyography (sEMG) (Sbriccoli et al., 2009), that assesses muscle activity and can be applied to determine the anaerobic threshold (Mello, Oliveira, \& Nadal, 2006), assessing neuromuscular diseases (Bandak, Amris, Bliddal, Danneskiold-Samsoe \& Henriksen, 2013), activation of motor units and muscle fatigue (Tartarisco et al., 2012) and the interaction between increased strength and increased range of recruitment of motor units (Qi, Wakeling, Green, Lambrecht, \& Ferguson-Pell, 2011). Considering the assessment of sEMG in cyclists, some studies have used this parameter to evaluate different cadences on activity performed using the bike (Dantas et al., 2009; Lucia, Hoyos, Perez, \& Chicarro, 2000; Nicolò et al., 2013; Takaishi, Yasuda, \& Moritani, 1994).

Longitudinal studies performed on a cycle ergometer with trained cyclists and untrained volunteers reveal cyclists have a greater preference for higher cadences (90-100 rpm) compared to untrained volunteers (50-60 rpm) (Dantas et al., 2009; Lucia et al., 2000; Takaishi, Yasuda, Ono, \& Moritani, 1996). These authors concluded that the preference was given due to the fact that although higher cadences promote greater metabolic demands, muscle fatigue was reduced (Dantas et al., 2009; Lucia et al., 2000; Takaishi et al., 1996).

Based on the considerations that HRV is an important noninvasive marker of sympathovagal modulation of heart rate related to exercise intensity and that sEMG is a valuable tool in the study of the muscle function during exercise, to our knowledge no studies have evaluated these variables in trained cyclists during dynamic exercise protocols on a cycle ergometer at different cadences. The importance of the contrast of the different cadences is to verify if the speed of pedaling can result in more pronounced responses of these variables between conditions.

Therefore, the aim of this study was to evaluate the behavior of heart rate (HR), cardiac modulation (through RMSSD and SDNN indices of HRV analysis) and sEMG of the vastus lateralis muscle in two protocols of dynamic exercise on a cycle ergometer at 60 and $80 \mathrm{rpm}$ in trained cyclists. The study hypothesis was that the protocol used in higher cadence $(80 \mathrm{rpm})$ promotes more pronounced responses of cardiac autonomic system and of myoelectric activity of the vastus lateralis compared to the protocol with the lower cadence $(60 \mathrm{rpm})$.

\section{METHOD}

\section{Study design and population}

This was an observational and retrospective study involving eight healthy men, all trained cyclists with high level of physical training for at least two years, with a minimum training frequency of 4-5 days per week and average duration of two hours per day. Exclusion criteria 
include: smokers, those who used drugs or alcohol, users of medications and athletes with musculoskeletal injuries in the lower limbs. Previously, the volunteers were informed about the purpose of the study and the methodology to be applied, to ensure evidence of the use of noninvasive tests that do not present a health risk. This study was approved by the local Institutional Research Ethics Committee and has been conducted according to the principles expressed in the Declaration of Helsinki. The procedure was explained to each subject separately and signed consent.

\section{General procedures and clinical evaluation}

All tests were performed at the same time of day (afternoon), thus avoiding possible interference of circadian variations in the behavior of the studied variables. The environmental conditions were controlled so that the temperature and relative humidity ranged from 20 to $23{ }^{\circ} \mathrm{C}$ and 40 to $60 \%$, respectively. All volunteers were instructed not to make strenuous efforts before the tests, looking to eat well, to not drink stimulants like caffeine and to get a good night's sleep the night before data collection. Clinical evaluation was performed by a cardiologist consisting of: 1) anamnesis, 2) anthropometric measurements (height and body weight), 3) resting 12-lead electrocardiography (ECG), 4) checking the blood pressure (BP) and heart rate (HR), 5) laboratory tests: complete blood count, glucose, urea, creatinine, uric acid, urinalysis, triglycerides, total cholesterol and fractions (LDL-cholesterol, HDL-cholesterol and VLDL-cholesterol) and 6) symptom-limited exercise test on a cycle ergometer.

The volunteers underwent three experimental sessions of exercise on an electromagnetic cycle ergometer on nonconsecutive days, with intervals of at least one week between sessions, involving three experimental procedures adequately standardized, being:

\section{Symptom-limited exercise testing}

The exercise test was performed by a cardiologist to evaluate the clinical and functional aspects of the cardiovascular system, as well as to detect possible changes that indicate against the participation of volunteers in research. A test was performed in the sitting position on an electromagnetic braked cycle ergometer (Quinton Corival 400, Groninger, Netherlands) at an initial power of $25 \mathrm{~W}$ for a warm-up period of 2 minutes. Subsequently, every three minutes, an increment of load of 50 $\mathrm{W}$ was applied until an intensity of $225 \mathrm{~W}$ was reached; from this load, the increments were 25 $\mathrm{W}$ until exhaustion or development of signs and limiting symptoms. During the testing, the volunteers were continuously monitored in leads MC5, DII and V2 (MC5: with the negative electrode at the apex of manubrium, positive at the fifth left intercostal space and neutral in the fifth right intercostal space; DII: with negative electrode at the apex of manubrium, positive at the sixth left intercostal space and neutral in fifth right intercostal space; V2: with positive electrode in the fourth left intercostal space). The HR, BP and ECG were recorded before the testing, at the end of 30 s at each power output, and in the recovery period $(1,3,6$ and 9 minutes).

\section{Dynamic exercise testing in continuous steps on a cycle ergometer at 60 and $80 \mathrm{rpm}$ (DETC- 60 and DETC-80)}

The volunteer was instructed to remain seated on the cycle ergometer at rest for 1 minute. The test started with an intensity of $25 \mathrm{~W}$ for 2 minutes and then a load was applied of approximately $30 \%$ of the median load reached in the test corresponding to symptom-limited exercise testing. From this, the increase of intensity led to $20 \mathrm{~W}$ every minute until physical exhaustion. Randomly, these protocols were performed with the aim of analyzing variability of $\mathrm{R}-\mathrm{R}$ intervals obtained beat by beat in dynamic exercise testing and to evaluate the muscle activity in relation to the intensities applied during exercise at different cadences (60 and 80 rpm).

\section{Variables collected during the DETC-60 and DETC-80}

$R-R$ intervals and $H R$ :

Electrocardiographic signals obtained were processed through an analogue to digital 
converter Lab PC + (National Instruments, CO). HR was obtained from the calculation of R-R intervals through specific software (Silva et al., 1994) that allowed the storage and handling of files for later analysis. Monitoring HR occurred during DETC-60 and DETC-80 and 1 minute in active recovery in which the volunteer remained pedaling at an intensity of $25 \mathrm{~W}$ followed by passive recovery (1 minute).

\section{Surface electromyography (sEMG):}

We used a computerized 16-channel electromyography (Lynx Electronic Technology) and a program of signal acquisition (AqDados5). The sEMG signals were processed in digital bandpass filter with cutoff frequency of $20 \mathrm{~Hz}$ and $500 \mathrm{~Hz}$, and that each experiment were calibrated with a gain of 1000 , being 20 times the electrode and 50 times the plate to obtain a reference point of calibration according to the manufacturer's manual.

Initially with the volunteer in the supine position were measured the dominant leg and subsequently trichotomy, abrasion and cleaning the skin with alcohol was performed for the placement of electrodes to capture the sEMG. The electrodes were placed at half the distance between the greater trochanter and lateral femoral condyle, coupled by a specific terminal device adapted active differential, which is responsible for the differentiation between the two poles of the electrodes.

The sEMG was recorded from the vastus lateralis muscle that was chosen because it is a large superficial muscle and actively involved in leg extension when the exercise is performed on a cycle ergometer. Moreover, it is frequently studied and often mentioned in studies using the cycle ergometer (Nagata, Muro, Moritani \& Yoshida, 1981; Viitasalo, Luhtanen, Rahkila \& Rusko, 1985).

\section{Data Analysis}

Analysis of heart rate variability:

HRV was analyzed with statistical measures in the time domain. The square root of the difference in the sum of squares between $R-R$ interval on the record divided by the determined time minus one (RMSSD), was analyzed to be an important index of HRV analysis which provided information on parasympathetic heart modulation and, the standard deviation of total normal R-R intervals (SDNN) was analyzed to be an index that represents the total HRV (cardiac sympathetic and parasympathetic modulation) (Task Force of the European Society of cardiology, 1996).

\section{Analysis of the electromyographic signal:}

Initially electromyographic signals obtained from the vastus lateralis (VL) of each volunteer during DETC-60 and DETC- 80 were separated by application of AqDados 5:03 (LYNX Electronic Technology) were divided into two parts: one containing signals to the load of $25 \mathrm{~W}$, and another section related to load increments, except for intensity of $25 \mathrm{~W}$, by the end of each protocol individually.

All sections separated were converted to ASCII format using the software AqDados 4.0 (LYNX Electronic Technology). Initially the sections were separated into windows containing 100 samples and were subsequently calculated the root mean square (RMS) of amplitude of the sEMG signal $(\mu \mathrm{V})$ of each windowing and RMS value of the electromyographic amplitude signal $(\mu \mathrm{V})$ corresponding to the entire signal.

From a new section containing only information about the RMS of the period of muscle contractions, each signal was divided in 256 consecutive samples which were analyzed in the RMS and Median Frequency (MF) specific of each window, and the values of the latter was obtained by fast Fourier transform (FFT) squared.

\section{Statistical Analysis}

Based on RMSSD values obtained with a previous pilot study, the sample size was determined using $G^{*}$ Power 3.1.13 for windows and for the current study, providing sufficient statistical power $(\beta=0.8)$, was estimated to be seven subjects. Data distribution was verified by the Shapiro-Wilk test and, the paired Student $t$ test was used to compare the variable obtained during symptom-limited exercise test at rest and peak condition. Friedman test with post-hoc of Dunn was used to compare the HR, RMSSD, 
SDNN and RMS in different effort intensities during protocols with different cadences and Wilcoxon matched pairs test was used to compare the values at the same intensity at different cadences. Spearman correlation was applied to verify the relationship between the responses of sEMG and HRV (RMSSD and SDNN) during exercise test. The probability of Type 1 error occurrence was established at $5 \%$ for all tests $(\alpha=0.05)$. Data were analyzed using the Statistica for Windows software release 5.1 (StatSoft, Inc, Tulsa, OK, USA).

\section{RESULTS}

The age, anthropometric characteristics, the level of physical activity and the variables obtained during symptom-limited exercise test are presented in Table 1.

Table 1

Anthropometric characteristics, physical activity level and variables obtained at rest and at peak exercise during symptom-limited exercise testing.

\begin{tabular}{|c|c|c|}
\hline & \multicolumn{2}{|c|}{ Variables } \\
\hline \multicolumn{3}{|l|}{ Age and anthropometric } \\
\hline Age (years) & \multicolumn{2}{|c|}{$28 \pm 5$} \\
\hline Height (m) & \multicolumn{2}{|c|}{$1.80 \pm 0.28$} \\
\hline Weight (kg) & \multicolumn{2}{|c|}{$77 \pm 8$} \\
\hline BMI $\left(\mathrm{kg} / \mathrm{m}^{2}\right)$ & \multicolumn{2}{|c|}{$24 \pm 2$} \\
\hline \multicolumn{3}{|l|}{ Physical activity level (cycling) } \\
\hline Weekly frequency (day/week) & \multicolumn{2}{|c|}{$5.3 \pm 0.7$} \\
\hline Duration (h/training session) & \multicolumn{2}{|c|}{$3.4 \pm 2.6$} \\
\hline Practice time (years) & \multicolumn{2}{|c|}{$7.5 \pm 4.4$} \\
\hline Symptom-limited exercise testing & Rest & Peak \\
\hline Maximum Power (W) & _ & $281 \pm 22$ \\
\hline SBP (mmHg) & $116 \pm 10$ & $195 \pm 16^{*}$ \\
\hline DBP (mmHg) & $74 \pm 7$ & $67 \pm 19$ \\
\hline HR estimated (bpm) & - & $192 \pm 5$ \\
\hline HR obtained (bpm) & $72 \pm 12$ & $196 \pm 6^{*}$ \\
\hline RPP (bpm/mmHg) & $8352 \pm 120$ & $38220 \pm 96$ \\
\hline $\mathrm{VO}_{2}$ estimated $(\mathrm{ml} / \mathrm{kg} / \mathrm{min} \uparrow)$ & - & $53 \pm 3$ \\
\hline $\mathrm{VO}_{2}$ calculated $(\mathrm{ml} / \mathrm{kg} / \mathrm{min})$ & - & $48 \pm 7$ \\
\hline
\end{tabular}

Data expressed as mean and standard deviation. BMI = body mass index, SBP = systolic blood pressure, $\mathrm{DBP}=$ diastolic blood pressure, $\mathrm{HR}=$ heart rate, $\mathrm{RPP}=$ rate-pressure product, $\mathrm{VO}_{2}=$ oxygen consumption. $\uparrow \mathrm{VO}_{2}$ calculated by the formula: [(intensity in $\mathrm{W}$ x 12) + 300] / Weight (Balke \& Ware, 1959). *Significant difference compared to the rest condition (paired Student t-test).

As noted in the table, the volunteers were training for cycling with weekly frequency of 5.3 days per week, with duration of 3.4 hours per training session on average. In addition, some volunteers (4 individuals) were sporadically performing recreational activities such as running or swimming 1 or 2 times a week, lasting approximately one hour. In relation to symptomlimited exercise test, as expected, a significant increase was observed in HR and systolic blood pressure (SBP), and decrease in diastolic blood pressure (DBP) from rest to peak exercise.

In both protocols during continuous dynamic exercise testing (60 rpm and $80 \mathrm{rpm}$ ) the maximum intensity in common for all volunteers was $240 \mathrm{~W}$ and therefore the analysis of variable intensities between $25 \mathrm{~W}$ to $240 \mathrm{~W}$ was considered. The behavior of the HR with increasing intensity during the tests are shown in Figure 1, increasing with intensity in both protocols (DETC-60 and DETC-80), with a significant increase from $200 \mathrm{~W}$ in relation to $25 \mathrm{~W}$ in the protocol performed at $60 \mathrm{rpm}$ and from $180 \mathrm{~W}$ in the protocol performed at $80 \mathrm{rpm}$. There were no significant differences in HR between the protocols (Figure 1).

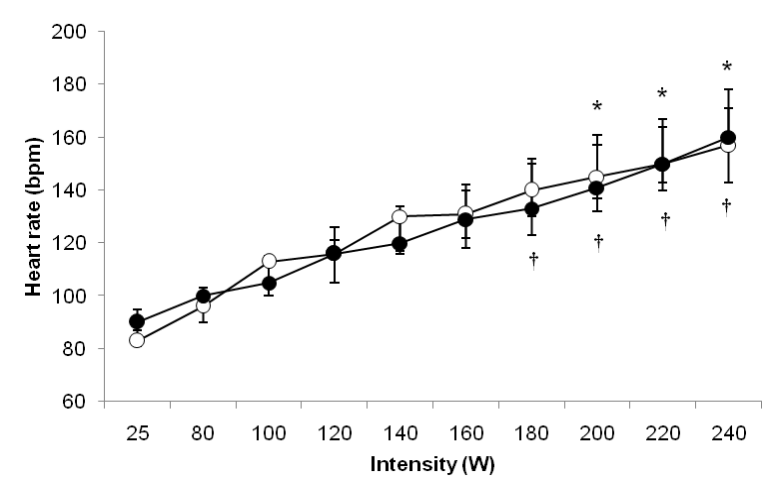

Figure 1. Data are presented as median with their respective 1 st and 3 rd quartiles. Behavior of heart rate during dynamic exercise testing in continuous steps on a cycle ergometer at $60 \mathrm{rpm}$ (open circle) and $80 \mathrm{rpm}$ (closed circle). * Significant difference in relation to the intensity of $25 \mathrm{~W}$ for cadence of $60 \mathrm{rpm}$; $\dagger$ Significant difference in relation to the intensity of $25 \mathrm{~W}$ for cadence of $80 \mathrm{rpm}$ (Friedman test). No difference $(p>0.05)$ between the cadences at the same intensity (Wilcoxon matched pairs test).

Regarding the behavior of HRV during the two protocols (DETC-60 and DETC-80), the RMSSD and SDNN values at each exercise intensity was shown in Figure 2 (A and B, respectively), with progressive reduction observed mainly in the first five exercise 
intensities (25W, 80W, 100W, 120W and 140W) followed by stabilization in response until the maximum intensity for all volunteers (240W). In the protocol performed at $60 \mathrm{rpm}$ significant reduction was observed from $160 \mathrm{~W}$ up to the last intensity of protocol $(240 \mathrm{~W})$ in relation to the initial intensity (25W) for both RMSSD as for SDNN. In the cadence of $80 \mathrm{rpm}$ significant reduction was observed from $140 \mathrm{~W}$ in relation to $25 \mathrm{~W}$, also in RMSSD and SDNN. When compared the intensities between the different cadences (60 rpm vs $80 \mathrm{rpm}$ ), there was no significant difference between them (both in RMSSD and SDNN).
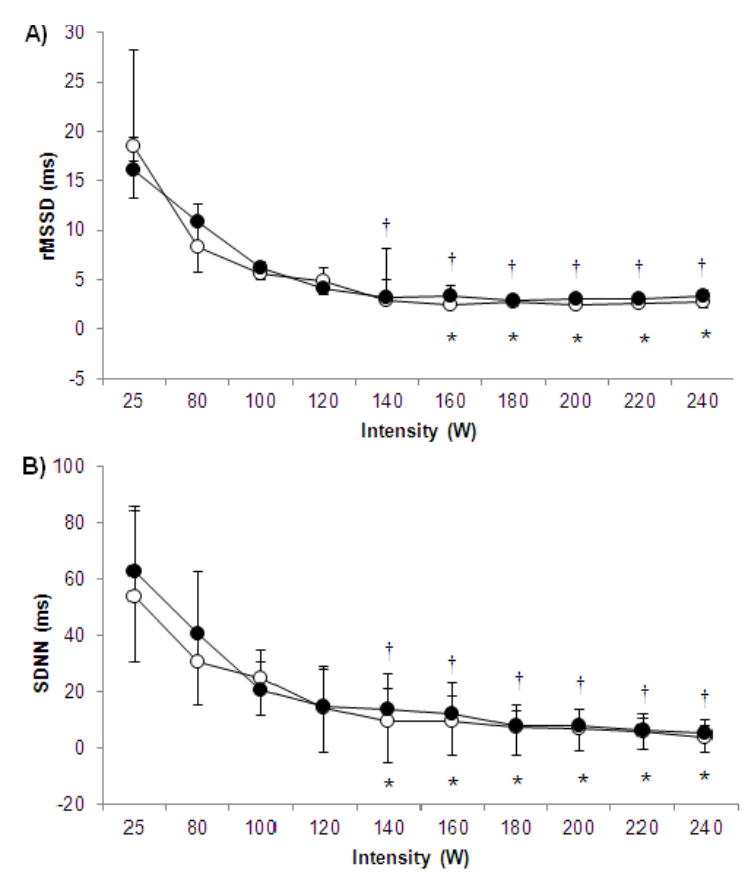

Figure 2. Data are presented as median with their respective 1 st and 3rd quartiles. (A) behavior of rMSSD (square root of the difference in the sum of squares between R-R interval on the record, divided by the determined time minus one) and (B) SDNN (standard deviation of R-R intervals) of volunteers during dynamic exercise testing in continuous steps on a cycle ergometer at $60 \mathrm{rpm}$ (open circle) and $80 \mathrm{rpm}$ (closed circle). *Significant difference in relation to the intensity of $25 \mathrm{~W}$ for cadence of $60 \mathrm{rpm}$; †Significant difference in relation to the intensity of $25 \mathrm{~W}$ for cadence of $80 \mathrm{rpm}$ (Friedman test). No difference ( $p>0.05$ ) between the cadences (60 vs $80 \mathrm{rpm}$ ) at the same intensity (Wilcoxon matched pairs test).

Regarding the behavior of RMS signal of the sEMG, it was observed that in both protocols (DETC-60 and DETC-80) there was a progressive increase in values with increasing intensity of cycle ergometer, which is significantly higher from $180 \mathrm{~W}$ in relation to $25 \mathrm{~W}$, both in the protocol performed at $60 \mathrm{rpm}$ and $80 \mathrm{rpm}$ (Figure 3).

When the values of HRV were correlated with electromyography of the vastus lateralis through RMSSD and SDNN with RMS of the SEMG, moderate and negative correlations, according to the classification of Cohen (1988), were found in both indices of HRV for both protocols performed at $60 \mathrm{rpm}$ (RMSSD with RMS: $\mathrm{r}=$ $0.42, \mathrm{p}=0.03$; SDNN with RMS: $\mathrm{r}=-0.45$, $\mathrm{p}=0.01$ ) and at $80 \mathrm{rpm}$ (RMSSD with RMS: $\mathrm{r}=-$ $0.47, p=0.02$; SDNN with RMS: $r=-0.49$, $\mathrm{p}=0.01)$.

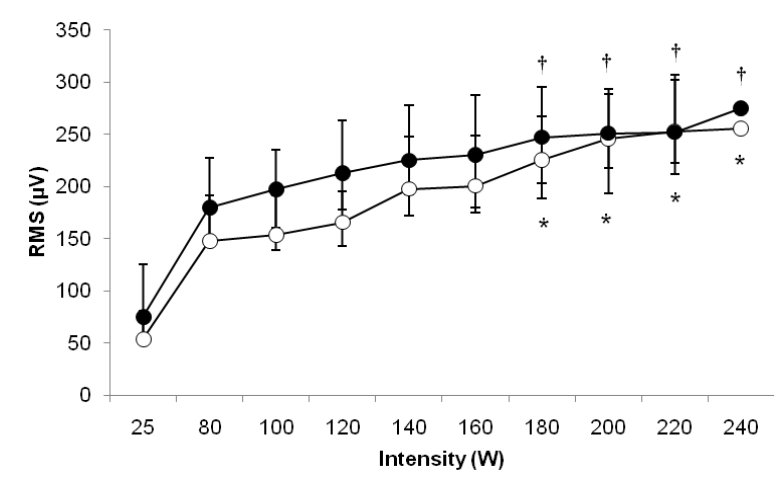

Figure 3. Data are presented as median with their respective 1st and 3rd quartiles. Behavior of the amplitude of electromyography signal as root mean square (RMS) values of volunteers during dynamic exercise testing in continuous steps on a cycle ergometer at $60 \mathrm{rpm}$ (open circle) and $80 \mathrm{rpm}$ (closed circle). * Significant difference in relation to the intensity of $25 \mathrm{~W}$ for cadence of $60 \mathrm{rpm}$; †Significant difference in relation to the intensity of $25 \mathrm{~W}$ for cadence of $80 \mathrm{rpm}$ (Friedman test). No difference ( $p>0.05)$ between the cadences at the same intensity (Wilcoxon matched pairs test).

\section{DISCUSSION}

The main findings of the present study were that there was no difference in the responses of HR, HRV and sEMG when compared the same power output between the protocols of dynamic exercise in continuous steps performed at $60 \mathrm{rpm}$ and $80 \mathrm{rpm}$. While the primary hypothesis of the study was not supported the protocol performed with a higher cadence $(80 \mathrm{rpm})$ promoted the earliest responses of $\mathrm{HR}$ and cardiac parasympathetic modulation in relationship to the protocol with lower cadence (60 rpm). Furthermore, there was correlation between the 
cardiac autonomic modulation and surface electromyography responses.

With regard to the characteristics of individuals, all had a high level of physical training which is specific to the type of exercise proposed in this study, cycling, reaching relatively high values of intensity at peak effort of symptom-limited exercise testing (average of 281 $\mathrm{W})$. Moreover, subjects reached a predicted maximal $\mathrm{HR}$ and were close to $\mathrm{VO}_{2}$ max predicted by age (Table 1). The weekly frequency and duration of training were similar between subjects, which provide increased reliability in relation to the homogeneity of the sample. It is worth noting that no subject was a smoker, drank alcohol or ingested any medication, in which factors could influence the responses of HR and HRV (parasympathetic and sympathetic modulation) during exercise testing.

As for the progressive increase in HR with increasing intensity during the protocols performed at different cadences, this behavior is well described in the literature as being the withdrawal of the vagal component active in the sinus node (Alonso et al., 1998) mainly in the initial intensities of the exercise protocol, a result that was verified in this study by the progressive reduction of the RMSSD index until stabilization of vagal modulation at a given power output. Similarly, the SDNN index that reflects the HRV total (sympathetic and parasympathetic modulation) also showed the same behavior in both protocols, possibly due to vagal withdrawal (Marães et al., 2005; Simões et al., 2013).

In a study by Alonso et al. (1998) in healthy young men with dynamic exercise type continuous steps and using the standard deviation of R-R intervals, the authors reported that the decrease of HRV (parasympathetic index) is more marked after AT intensity. The authors also reported that possibly the decreased and subsequent stabilization of the HRV is associated with increased sympathetic contribution after AT.

Simões et al. (2014) used a discontinuous incremental protocol in cycle ergometer in healthy elderly subjects and found a significant correlation between blood lactate and heart rate variability (through index RMSSD) on AT determination. The authors also found marked reduction of parasympathetic index (RMSSD), such as an index that represents the total HRV (RMSM), at lower intensities of exercise followed by stabilization of the variable that occurred after the intensity corresponding to AT.

With respect to RMS signal of sEMG, the significant increase from $180 \mathrm{~W}$ in both protocols with different cadences (DETC-60 and DETC80) may reflect changes in the type of muscle fibers recruited from this intensity that reflect adaptations of the muscular and metabolic systems in an attempt to maintain the continuity of physical activity and the homeostasis of the systems. Studies have reported that trained cyclists have a lower percentage of type II fibers and a higher percentage of type I fibers in the vastus lateralis (Coyle, Sidossis, Horowitz, \& Beltz, 1992; Hopker et al., 2013) with the type I fibers being initially recruited followed by the gradual recruitment of the type II fibers (Gamet, Duchene, Garapon-Bar, \& Goubel, 1993). Hansen, Andersen, Nielsen, and Sjøgaard (2002) studying recreational cyclists, observed a relationship between the preference of cadences with the percentage of certain types of muscle fibers and individuals with higher percentages of type I fibers chose higher cadences; lower cadences were chosen by individuals with higher percentages of type II fibers .

The most preferred cadences by cyclists are around 85 to $95 \mathrm{rpm}$, while the most economical cadences are approximately between 55 and 60 rpm (Dantas et al., 2009; Takaishi et al., 1996). Vercruyssen et al. (2002) found that the speeds between 80 and $90 \mathrm{rpm}$ have less relationship to muscle fatigue and also higher cadences are associated with greater neuromuscular economy where the volunteers had preference for higher cadences. However, the preferred cadences of cyclists do not coincide with lower oxygen consumption, but instead with less development of muscle fatigue (Takaishi et al., 1996; Vercruyssen \& Brisswalter, 2010).

Studies involving different cadences in triathletes concluded that higher cadences in cycling can contribute to a better performance in the subsequent phase of the triathlon, the foot race. However, this "great" cadence generates a 
decrease of energy during cycling and consequently the race (Vercruyssen et al., 2002). In higher cadences there is reduced force applied to the pedal ergometer, which consequently decrease fatigue due to reduced recruitment of type II fibers and increased recruitment of type I fibers (Lucia et al., 2000).

In this study it was not possible to verify the loss of linearity of the signal amplitude of RMS of EMG in both protocols as there was no difference in values between the DETC-60 and DETC-80, so maybe the difference between the cadences chosen was too small to reflect changes between the variables analyzed in this study. However, we observed correlation between the cardiovascular and muscular responses front of protocols which reflects an interaction between these systems, and possibly peripheral factors such as increased recruitment of motor units, such as the type II fibers, the possible increase of catecholamine's in blood circulation and reduction in blood flow due to increased intramuscular pressure when the muscle tension increase significantly (detection by peripheral receptors called mechanoreceptors and metaboreceptors fibers - type II and type IV) transmit this information to the central nervous system triggering responses through cardiac vagal and sympathetic efferent modulations (Fadel \& Raven, 2012; Mitchell, 1990; Mitchell, 2013; Rowell \& O'Leary, 1990; Rowell, 1992).

Although this study did not have as its objective the determination of AT, our results of HR, HRV and sEMG reflect changes in cardiac autonomic control and muscular system that at any given time of the exercise protocol may indicate adjustments of the cardiovascular and muscle systems in an attempt to meet the increased demand in higher intensities of exercise.

No difference in the responses of sEMG, HR and HRV were seen when compared to the same power output between the protocols of dynamic exercise in continuous steps performed at $60 \mathrm{rpm}$ and $80 \mathrm{rpm}$, indicating that the highest cadence does not promote different responses both in relation to the electrical activity of the muscles as to cardiac autonomic modulation, which suggests that even the activity performed at the highest cadence possibly not cause early muscle fatigue, and that in relation to cardiac autonomic modulation, the cardiac protection provided by vagal modulation is the same in both cadences.

Some limitations of this study must be considered, such as, metabolic and ventilatory variables were not evaluated using ergospirometry. Moreover, blood lactate measured simultaneously with cardiac autonomic modulation and surface electromyography responses were not collected allow us to determine the intensity corresponding to the AT and thus relate the behavior of aerobic to anaerobic predominance in both protocols during dynamic exercise.

\section{CONCLUSION}

Based on the results of this study, we concluded that no differences in surface electromyography and cardiac autonomic responses are apparent when comparing the same power output between the protocols during dynamic exercise in continuous steps performed on a cycle ergometer at $60 \mathrm{rpm}$ and $80 \mathrm{rpm}$. We suggest that higher cadences (which are the preferred cadence for these athletes) may not promote significant changes that could result in fatigue and early interruption of effort. It also is possible, that the cardiac protection provided by vagal modulation is the same in both cadences.

\section{Acknowledgments:}

The authors would like to thank the Coordenação de Aperfeiçoamento de Pessoal de Nível Superior (CAPES) for the financial support to the Master's scholarship grants to APGU.

\section{Conflict of interest:}

Nothing to declare

\begin{tabular}{l}
\hline Funding \\
This study was funded by CAPES (PNPD \\
$23038.006927 / 2011-92)$.
\end{tabular}

\section{REFERENCES}

Alonso, D. O., Forjaz, C. L., Rezende, L. O., Braga, A. M., Barreto, A. C., Negrão, A. C. \& Rondon, M. U. (1998). Heart rate response and its variability during different phases of maximal graded exercise. Arquivos Brasileiros de Cardiologia, 
71(6), $\quad 787-792 . \quad$ doi: $10.1590 /$ S0066782X1998001200008

Balke, B., Ware, R. W (1959). An experimental study of physical fitness of air force personnel. United States Armed Forces Medical Journal, 6(10), 675688.

Bandak, E., Amris, K., Bliddal, H., Dannskiold-Samøe, B. \& Henriksen, M. (2013). Muscle fatigue in fibromyalgia is in the brain, not in the muscles: a case-control study of perceived versus objective muscle fatigue. Annals of Rheumatic Diseases, 72(6), 963-966. doi: 10.1136/annrheumdis2012-202340.

Catai, A. M., Chancon-Mikahil, M. P. T., Martinelli, F. S., Fort, V. A., Silva, E., Golfetti, R., ... Gallo Jr, L. (2002). Effects of aerobic exercise training on heart rate variability during wakefulness and sleep and cardiorespiratory responses of young and middle aged healthy men. Brazilian Journal of medical and Biological Research, 35(6), 741752. doi: 10.1590/S0100-879X2002000600016.

Coyle, E. F., Sidossis, L. S., Horowitz, J. F. \& Beltz, J. D. (1992). Cycling efficiency is related to the percentage of type I muscle fibers. Medicine \& Science in Sports \& Exercise, 24(7), 782-788. doi:10.1249/00005768-199207000-00008.

Cohen, J. (1988). Statistical power analysis for the behavioral sciences. Hillsdale, NJ, Erlbaum, 2nd edition: Lawrence Erlbaum Associates.

Dantas, J. L., Smirmaul, B. P., Altimari, L. R., Okanko, A. H., Fontes, E. B., Camata, T. V. \& Moraes, A. C. (2009). The efficiency of pedaling and the muscular recruitment are improved with increase of the cadence in cyclists and non-cyclists. Electromyography and Clinical Neurophysiology, 49(6-7), 311-319.

Di Michele, R., Gatta, G., Leo, A. D., Cortesi, M., Andina, F., Tam, E., Boit, M. D. \& Merni, F. (2012). Estimation of the anaerobic threshold from heart rate variability in an incremental swimming test. Journal of Strength and Conditioning Research, 26(11), 3059-66. doi: 10.1519/JSC.0b013e318245bde1.

Fadel, P. J. \& Raven, P. B (2012). Human investigations into the arterial and cardiopulmonary baroreflexes during exercise. Experimental Physiology, 97(1), 39-50. doi: 10.1113/expphysiol.2011.057554.

Gamet, D., Duchene, J., Garapon-Bar, C. \& Goubel, F. (1993). Surface electromyogram power spectrum in human quadriceps muscle during incremental exercise. Journal of Applied Physiology, 74(6), 2704-2710.

Hansen, E. A., Andersen, J. L., Nielsen, J. S. \& Siøgaard, G. (2002). Muscle fiber type, efficiency, and mechanical optima affect freely chosen pedal rate during cycling. Acta Physiologica Scandinavica, 176(3), 185-194. doi: 10.1046/j.1365201X.2002.01032.x

Hopker, J. G., Coleman, D. A., Gregson, H. C., Jobson, S. A., Von de Haar, T., Wiles, J. \& Passfield, L. (2013). The influence of training status, age, and muscle fiber type on cycling efficiency and endurance performance. Journal of Applied Physiology, 115(15), 723-729. doi:10.1152/japplphysiol.00361

Longo, A., Ferreira, D. \& Correia, M. J. (1995). Variabilidade da frequência cardíaca. Revista Portuguesa de Cardiologia, 14(3), 241-262.

Lucia, A., Hoyos, J., Pérez, M. \& Chicharro, J. L. (2000). Heart rate and performance parameters in elite cyclists: a longitudinal study. Medicine \& Science in Sports \& Exercise, 32(10), 1777-1782.

Marães, V.R., Silva, E., Catai, A.M., Novais, L.D., Moura, M.A., Oliveira, L., \& Gallo Jr, L. (2005). Identification of anaerobic threshold using heart rate response during dynamic exercise. Brazilian Journal of Medical and Biological Research, 38(5), 731-735. doi: 10.1590/S0100$879 \times 2005000500010$.

Mello, R. G., Oliveira, L. F. \& Nadal, J. (2006). Detection of the anaerobic threshold by surface electromyography. Annual International Conference of the IEEE Engineering in Medicine and Biology Society, 1, 6189-6192. doi: 10.1109/IEMBS.2006.260619.

Mitchell, J. H. (1990). Neural control of the circulation during exercise. Medicine \& Science in Sports \& Exercise, 22(2), 141-154.

Mitchell, J. H. (2013). Neural circulatory control during exercise: early insights. Experimental Physiology, 98(4), 867-878. doi: 10.1113/expphysiol.2012.071001.

Nagata, A., Muro, M., Moritani, T. \& Yoshida, T. (1981). Anaerobic threshold determination by blood lactate and myoelectric signals. Japanese Journal of Physiology, 31(4), 585-597.

Nicolò, A., Bazzucchi, I., Lenti, M., Haxhi, J., Scotto Di Palumbo, A. \& Sacchetti, M. (2013). Neuromuscular and metabolic responses to highintensity intermittent cycling protocols with different work-to-rest ratios. International Journal of Sports Physiology and Performance, 9(1), 151-160. doi: 10.1123/ijspp.2012-0289.

Qi, L., Wakeling, J. M., Green, A., Lambrecht, K. \& Ferguson-Pell, M. (2011). Spectral properties of electromyographic and mechanomyographic signals during isometric ramp and step contractions in biceps brachii. Journal of Electromyography \& Kinesiology, 21(1), 128135. doi: 10.1016/j.jelekin.2010.09.006.

Ribeiro, T. F., Cunha, A., Lourenço, G. C. D., Marães, V. R. F. S., Catai, A. M., Gallo Jr, L. \& Silva, E. (2000). Estudo da variabilidade da frequência cardíaca em dois voluntários de meia idade, um coronariopata e outro saudável: relato de caso. Revista da Sociedade de Cardiologia do Estado de São Paulo, 10(supl A), 1.

Rowell, L. B. \& O'leary, D. S. (1990). Reflex control of the circulation during exercise: chemoreflexes and mechanoreflexes. Journal of Applied Physiology, 69(2), 407-418. 
Rowell, L. B. (1992). Reflex control of the circulation during exercise. International Journal of Sports Medicine, 13(1), S25-S27.

Sales, M. M., Campbell, C. S., Morais, P. K., Ernesto, C., Soares-Caldeira, L. F., Russo, P, Simões, H. G. (2011). Noninvasive method to estimate anaerobic threshold in individuals with type 2 diabetes. Diabetology \& Metabolic Syndrome, 3(1), 1. doi: 10.1186/1758-5996-3-1.

Sbriccoli, P., Sacchetti, M., Felici, F., Gizzi, L., Lenti, M., Scotto, A. \& De Vito, G. (2009). Noninvasive assessment of muscle fiber conduction velocity during an incremental maximal cycling test. Journal of Electromyography \& Kinesiology, 19(6), 380-386. doi: 10.1016/j.jelekin.2009.03.008

Seluyanov, V. N., Kalinin, E. M., Pack, G. D., Mayevskaya, V. I. \& Konrad, A. N. (2011). Estimation of the anaerobic threshold from the data on lung ventilation and heart rate variability. Human Physiology, 37(6), 733-737. doi: 10.1134/S0362119711060132.

Silva, E., Catai, A. M., Trevelin, L. C., Guimarães, J. O., Silva Jr, L. P., Silva, L. M. P., ... Gallo Jr., L. (1994). Design of a computerized system to evaluate the cardiac function during dynamic exercise (abstract). Physics in Medicine and Biology, 39a, 409.

Silva, E., Oliveira, L., Catai, A. M., Ferreira Filho, P., Bérzin, F. \& Gallo Jr, L. (1999). Evaluation of electromyography activity and heart rate response to isometric exercise. The role played by muscular mass and type. Brazilian Journal of medical and Biological Research, 32(1), 115-120. doi: 10.1590/S0100-879X1999000100017.

Simões, R. P., Bonjorno Jr. J. C., Beltrame, T., Catai, A. M., Arena, R. \& Borghi-Silva, A (2013). Slower heart rate and oxygen consumption kinetic responses in the on- and off-transient during a discontinuous incremental exercise: effects of aging. Brazilian Journal of Physical Therapy, 17(1), 69-76. 10.1590/S141335552012005000056.

Simões, R. P., Castello-Simões, V., Mendes, R. G., Archiza, B., Dos Santos, D. A., Bonjorno Jr, J. C., ... Borghi-Silva, A. (2014). Identification of anaerobic threshold by analysis of heart rate variability during discontinuous dynamic and resistance exercise protocols in healthy older men. Clinical Physiology and Functional Imaging, 34(2), 98-108. doi:10.1111/cpf.12070.

Task Force of the European Society of cardiology and the North American Society of Pacing and Eletrophysiology. (1996). Heart rate variability. Standad of measurement, physiological interpretation and clinical use. Circulation, 93(5), 1043-1065.

Takahashi, A. C. M., Novais, L. D., Silva, E., Oliveira, L., Milan, L. A., Darezzo, F., ... Catai, A. M. (2005). Avaliação do controle autonômico da frequência cardíaca e determinação do limiar de anaerobiose em homens saudáveis e coronariopatas. Revista Brasileira de Fisioterapia, 9(2), 157-164.

Takaishi, T., Yasuda, Y. \& Moritani, T. (1994). Neuromuscular fatigue during prolonged pedaling exercise at different pedaling rates. European Journal of Applied Physiology and Occupational Physiology, 69(2), 154-158.

Takaishi, T., Yasuda, Y., Ono, T. \& Moritani, T. (1996). Optimal pedaling estimated from neuromuscular fatigue for cyclists. Medicine \& Science in Sports \& Exercise, 28(12), 1492-97. doi: 10.1097/00005768-199612000-00008.

Tartarisco, G., Billeci, L., Ricci, G., Volpi, L., Pioggia, G. \& Siciliano, G. (2012). A personal monitoring architecture to detect muscular fatigue in elderly. Neuromuscular Disorders, 22(sup. 3), S192-197. doi: 10.1016/j.nmd.2012.10.009.

Vercruyssen, F., Brisswalter, J., Hausswirth, C., Bernard, T., Bernard, O. \& Vallier, J. M. (2002). Influence of cycling cadence on subsequent running performance in triathletes. Medicine \& Science in Sports \& Exercise, 34(3), 530-536. doi: 10.1097/00005768-200203000-00022.

Vercruyssen, F. \& Brisswalter, J. (2010). Which factors determine the freely chosen cadence during submaximal cycling? Journal of Science and Medicine in Sport, 13(2), 225-231. doi: 10.1016/j.jsams.2008.12.631.

Viitasalo, J. T., Luhtanen, P., Rahkila, P. \& Rusko, H. (1985). Electromyographic activity related to aerobic and anaerobic threshold in ergometer bicycling. Acta Physiologica Scandinavica, 124(2), 287-293. doi: 10.1111/j.17481716.1985.tb07663. 